- Case Report

\title{
Unusual presentation of ciliary body melanoma - a case report
}

\author{
T Sarbajna ${ }^{1}$, P Lavaju ${ }^{1}$, M Agarwal $^{2}$, K Ahmad $^{3}$ \\ ${ }^{1}$ Department of Ophthalmology, ${ }^{2}$ Department of Pathology, ${ }^{3}$ Department of Radiodiagnosis \\ and Imaging \\ B P Koirala Institute of Health Sciences, Dharan, Nepal
}

\begin{abstract}
Background: Ciliary body melanoma rarely grows transclerally by direct extension and can metastasize locally into the orbit or conjunctiva. Objective: To report a case of ciliary body melanoma presenting clinically as a mass in the lacrimal gland region. Case: A40-year-old female presented to the ophthalmic out-patient department with the complaint of a swelling in the right upper and outer lid region for 4 years. Ocular examination revealed a phthisical right eye. A firm, non-tender mass was palpable in the temporal quadrant of the right supraorbital region. Ultrasonography showed retinal detachment of the right eye. The CT-scan of the orbit was reportedly suggestive of a right lacrimal gland mass. Superolateral transperiosteal orbitotomy was planned for excision of the lacrimal gland mass. Intraoperatively, a well defined mass arising from the supero-temporal region of the right globe was noticed. The procedure was converted to enucleation of the right eye. Histopathological examination of the mass showed features of ciliary body melanoma. Conclusion: Extrascleral extension of ciliary body melanoma can present as a mass in the lacrimal gland region.
\end{abstract}

Keywords: Ciliary body melanoma, lacrimal gland mass, superolateral transperiosteal orbitotomy, enucleation

\section{Introduction}

Ciliary body melanoma is a subtype of uveal melanoma. Uveal melanoma, which is the most common primary malignant intraocular tumor, is classified depending on the site of its origin. It is called anterior when the tumor arises in the iris, and posterior, when it arises in either the choroid or the ciliary body. ${ }^{1}$

Studies have shown that the incidence of primary intraocular melanoma is around 4.3-6 cases per 1 million population. Ciliary body melanoma is quite rare and represents about one tenth of all intraocular melanomas. ${ }^{2,3}$

Ciliary body melanoma usually remains intraocular. The usual site is behind the iris diaphragm, and it normally does not show extraocular extension. But since the eye has no lymphatic vessels, it can sometimes disseminate hematogenously and spread preferentially to the liver. ${ }^{4}$

Address for correspondence

Dr Tushar Sarbajna, MD

Department of Ophthalmology

BPKIHS, Dharan-18, Nepal

E-mail: tushar_sarbajna@yahoo.com
Less frequently, ciliary body melanoma can grow transsclerally, through emissary channels, and can metastasize locally especially into the orbit and conjunctiva. $^{5}$

Some ciliary body melanomas can show diffuse growth patterns and can extend around the entire circumference of the ciliary body. Such melanomas have a greater tendency to grow extrasclerally. ${ }^{6}$

We report a case of ciliary body melanoma presenting clinically as a mass in the lacrimal gland region.

\section{Case report}

A 40-year-old female presented to the ophthalmic outpatient department with the complaint of a swelling in the right upper and outer lid region for 4 years. The mass was initially pea sized and gradually progressed. It was initially painless but since past few months it was associated with pain. There was no history of trauma or forward bulging of the eye. There was a history of pain and redness in the right eye 7 years back leading to loss of vision. 
Ocular examination revealed a phthisical right eye with the visual acuity of no perception of light. A $20 \times 10$ $\mathrm{mm}$, firm, round, non-tender and smooth mass was palpated in the temporal quadrant of the right supraorbital region. Examination of the left eye was within normal limits.

Ultrasonography showed retinal detachment of the right eye. The CT-scan of the orbit showed a $21 \times 10 \mathrm{~mm}$ soft tissue density mass in the right lacrimal region abutting the superolateral aspect of right globe. A provisional diagnosis of lacrimal gland tumor was made. Systemic evaluation and investigations were within normal limits.

A superolateral transperiosteal orbitotomy for excision of the mass was planned under general anaesthesia. A curvilinear incision extending from the lateral side of the right eyebrow to $15 \mathrm{~mm}$ from the lateral canthus was made. The periosteum was elevated and the mass identified.

The lacrimal gland was found to be normal.A well defined, round and smooth mass measuring approximately $20 \times 10 \mathrm{~mm}$ was noticed arising from the supero-temporal aspect of the right globe. The operative procedure was changed to enucleation. Enucleation of the right eye was performed and the eyeball was subjected for histopathological examination.

Gross examination revealed a tumor originating from the superior aspect of the eyeball measuring $20 \mathrm{~mm}$ in the greatest diameter and occupying whole of the superolateral aspect of the eyeball. Cut surface of the tumor was solid and grey white with focal brownish discoloration.

Microscopic examination showed proliferation of tumor cells occupying whole of the eyeball at places arising from the ciliary body. The tumor cells comprised of round to spindle shaped cells and between them, there were dispersed melanin pigments alongwith few inflammatory cells.

The overall histomorphological features were those of malignant melanoma.

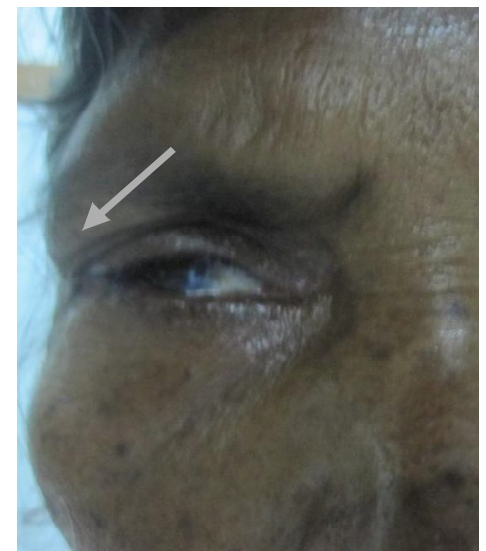

Figure 1: A $20 \times 10 \mathrm{~mm}$ swelling in the temporal quadrant of the right supraorbital region (arrow) and a phthisical right eye.

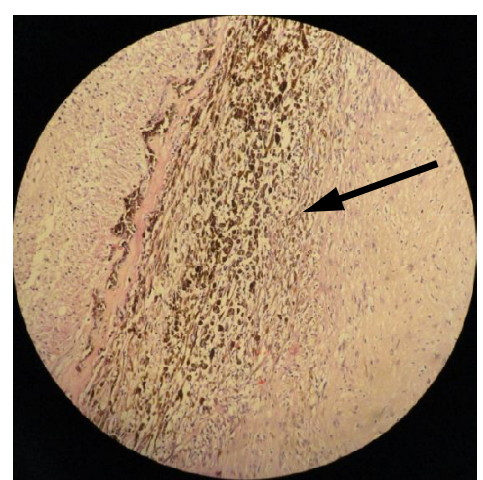

Figure 2: Malignant melanoma of the ciliary body composed of spindle shaped cells dispersed melanin pigments (arrow) characterised by vesicular chromatin visible to prominent eosinophilic nucleoli and moderate amount of cytoplasm (haematoxylin and eosin staining, magnification 40x).

The investigations done post-operatively (chest x-ray, ultrasonography of the abdomen) did not reveal any evidence of metastasis.

\section{Discussion}

Ciliary body melanomas are quite rare and are not recognised early because of their location. Thus, they can become quite large before being clinically diagnosed. ${ }^{7}$ They spread by direct extension, can metastasize locally, or can show generalised metastasis. ${ }^{8}$ Extrascleral extension occurs through the emissary canals ${ }^{5}$ and usually means a poor prognosis. ${ }^{9}$ A retrospective study in 1986 by Pach et al showed that out of 610 patients with ciliary body or choroidal melanoma, $50(8.2 \%)$ had extrascleral extension and the factors affecting survival were tumor size and its 
location. ${ }^{10}$ Cases of extraocular spread of ciliary body melanoma have been well documented. Saad et al in 1990 reported a case of ciliary body melanoma invading the optic nerve. The patient had initially been treated for uveitis, but following CT scan and ultrasound, a tumour was detected and the eye enucleated. Histopathological report showed that the tumour had invaded the optic nerve head via Cloquet's canal. ${ }^{11}$ Henna et al in 2007 reported an interesting case of ciliary body melanoma presenting as an extraocular metastasis in the temporalis muscle alongwith pigmented cells seeding the vitreous. ${ }^{12}$ Our case is an example of an unusual presentation of ciliary body melanoma. It shows how an extrascleral extension of ciliary body melanoma can clinically present as a lacrimal gland mass. In the case, the CT scan orbit findings were thought to be consistent with a lacrimal gland mass because of its location in the supero-lateral orbit. It was only intraoperatively that an intraocular mass with an extrascleral extension was visualised and the operative plan converted from superolateral transperiosteal orbitotomy to enucleation. Histopathological findings of the enucleated eye was consistent with that of ciliary body melanoma. This case illustrates an interesting and unusual clinical presentation of a malignant melanoma of the ciliary body with extrascleral extension.

\section{Conclusion}

Extrascleral extension of a ciliary body melanoma can present clinically as a mass in the lacrimal gland region.

\section{References}

1. Duke-Elder S, Perkins ES. Cysts and tumors of the uveal tract. System of Ophthalmology. St. Louis:CV Mosby Company 1977; 9:843-911.

2. Singh A D, Topham A. Incidence of uveal melanoma in the United States: 1973-
1997. Ophthalmology 2003; 11:956-961.

3. Egan K M, Seddon J M, Glynn R J, et al. Epidemiologic aspects of uveal melanoma. Surv Ophthalmol 1988; 32:239-251.

4. Toivonen P, Mäkitie T, Kujala E, et al. Microcirculation and tumor infiltrating macrophages in choroidal and ciliary body melanoma and corresponding metastases. Invest Ophthalmol Vis Sci 2004; 45(1):1-6.

5. Wilcox TK. Case report: ciliary body melanoma with extrascleral extension. J Am Optom Assoc 1991;62:529-532.

6. Seregard S. Massive intracorneal invasion of a ciliary body melanoma. Acta Ophthalmol (Copenh) 1994;72:257-259.

7. Jain S S, Thomas S, Motware S A, Hamidani A. Malignant melanoma of ciliary body: A case report. Indian J Ophthalmol 1999;47:255-6.

8. Pavan-Langston D. Uveal tract: iris, ciliary body and choroid. Manual of Ocular Diagnosis and Therapy. 4th ed. Boston; Little, Brown and Company 1996; pp 181-227.

9. Gragoudas ES, Egan KM, Seddon JM, et al. Survival of patients with metastases from uveal melanoma. Ophthalmology 1991;98 (3):383-9.

10. Pach JM, Robertson DM, Taney BS, et al. Prognostic factors in choroidal and ciliary body melanomas with extrascleral extension. Am J Ophthalmol 1986;101 (3):325-31.

11. Al-Haddab S, Hidayat A, Tabbara K F: Br J Ophthalmol 1990;74:123-124.

12. Anu Liisa Oittinen H, O’Shaughnessy M, Keohane C: J Clin Pathol 2007;60(7):834-835. 